\title{
DETERMINATION OF THE INFLUENCE OF CALIBRATION SPHERE PARAMETERS ON THE RESULTS OF ARTICULATING PROBE HEAD QUALIFICATION
}

\author{
Maciej Gruza' , Adam Gąska', Piotr Gąska', Wiktor Harmatys' ${ }^{1}$, Jerzy Sładek¹ \\ 1 Laboratory of Coordinate Metrology, Cracow University of Technology, al. Jana Pawła II 37, 31-864 Cracow, \\ Poland, e-mail: wiktorharmatys@gmail.com, gruzam@interia.pl, piotr.gaska@pk.edu.pl, agaska@mech. \\ pk.edu.pl, sladek@mech.pk.edu.pl
}

Received: 2017.11.27

Accepted: 2018.05.13

Published: 2018.06.01

\begin{abstract}
The qualification of probe heads before the measurements performed with their usage is now a standard procedure. It is used for correction of probe head's and measuring tip systematic errors. The calibration sphere, which is used for this process, should have adequately small form errors. However, it is not always possible to use a highly accurate spheres and it is a common practice to use a sphere that was provided by the manufacturer of the CMM for a long time. This is why, the authors have tried to answer the question about the impact of the calibration sphere inaccuracies on the results of probe head qualification process.
\end{abstract}

Keywords: articulating probe head, calibration, accuracy.

\section{INTRODUCTION}

The industrial and technological growth requires accurate measuring techniques. The increasing speed of production process with simultaneous cost reduction pose new challenges for both the precision engineering industry and scientists working on the improvement of the measuring tools properties. New trends can be observed also in case of Coordinate Measuring Machines (CMMs). The main advantages of CMMs are well known, to mention only: measuring process automation, versatility and high accuracy. Nevertheless, there are still some areas to which efforts can be directed in order to ameliorate the CMM performance. One of the major difficulties is assurance of stable environmental conditions during measurements, the issue still largely unsolved in case of production lines and large industrial halls [3]. This problem is crucial for further development of in-process measurement realized using CMMs which would be a part of production line. The reduction of cost that would be ob- tained utilizing such a solution are clearly visible. Another issues connected with CMMs measurements include the need of experienced and well trained personnel which would ensure the proper usage of devices [9], and the problem of periodical machines accuracy control, which is essential to keep the machine's indications reliable $[1,4]$.

The indications of CMMs have to be referred to the base unit of length in the International System of Units (SI) what is achieved by machine calibration which ensures traceability. Assumption is quite simple, however, the way of determining consistency is usually complex. Each country has an appropriate institution responsible for traceability assurance. In Poland traceability at international level is ensured by the Central Office of Measures (GUM) while its assurance at national level is realized mostly by accredited calibration laboratories which control the indications of measuring tools and calibrate artifacts used in industry and science.

It should be noted that the calibration process is done for CMMs only over the relatively 
long time intervals (for example once a year) so it is not very sensitive to changes in the metrological properties of the machine. At least partial information about machine traceability on the daily basis can be obtained through the probe head qualification process $[5 \div 8]$. The procedure of qualification is very similar for different types of CMMs and for various machine's producers. It involves the measurements of material spherical standard, precisely manufactured reference ball. Such standards have to fulfil special requirements: appropriate diameter depending on the size of qualifying tip ball, but not extending $30 \mathrm{~mm}$, to minimize the influence of machine kinematics; very small form deviation, expressed in tenth of micrometres. The main principle of qualification process is the assumption that the reference ball is perfect (its form deviation equals to zero or is negligible from the perspective of the accuracy of the qualified probe head). By measuring reference ball CMM determines its current level of accuracy with respect to standard ball calibration method. The results from this measurement are recorded and then during actual measurements each measured point is corrected by the corresponding vector resulting from the qualification algorithm (radial correction). That is the reason why reference ball maintenance and appropriate storage are essential for proper CMM functioning. It is also worth mentioning that actions like probe tip cleaning and fixing taken prior to the qualification are equally important because omissions in these areas can lead to inaccuracies in radial correction. The qualification procedure itself is predefined depending on the used metrological software and the configuration of probe head.

To illustrate the importance of qualification, it can be said that during the procedure some phenomena associated with probe head functioning are taken into account in the overall measurement result. That include: deflection of the stylus during probing, plastic deformation of the tip ball or the probe pre-travel $[5,7]$. All of the above-mentioned phenomena depend on parameters related to probe configuration, geometry and parameters that describe the probing process as probing speed or acceleration. As can be seen, qualification should be repeated if any of the mentioned parameters change. Moreover, it should be borne in mind that shape deviations of the reference ball are transmitted onto the tip ball. In other words, the probe head measures with the accuracy of the qualification process and the accuracy of standard sphere calibration. Of course, the accuracy of the whole system depends also on other factors, such as geometric errors related to machine construction and temperature compensation. However, qualification is a very important part of overall machine accuracy, and it is also the first test of correctness of machine performance.

\section{QUALIFICATION OF THREE-AXIS COORDINATE MEASURING MACHINES}

Most of CMMs currently used in industry are capable of measurements performed in standard three axis mode. They acquiring the coordinates of a point, measured along the three mutually perpendicular axes that forms the basic Cartesian system of machine and determines directions along which the machine parts can shift. Depending on the used construction solutions, CMMs can be divided into several groups, with portal machines being most popular one. ZEISS WMM 850 machine used in described research is an example of a CMM belonging to this group. The system responsible for point coordinates acquisition is a probe head. Depending on the head operation principle they can be divided into: touch-trigger and measuring ones. The main task of probe heads from the first group is signalization of the contact occurrence between tip ball and measuring surface. The second group is additionally capable of measuring the probe deflection during contact process what generally contributes to the better accuracies obtained by such probes.

The calibration process on 3 axis machines is relatively fast, and the path of the tip and the head can be easily predicted. The qualification is done automatically and the operator has no possibility of interfering with the process except stopping it. The first step of process involves determination of the reference ball position in measuring volume of machine (coordinates of the ball centre). In the next part the ball is measured in the number of points distributed regularly on the: upper hemisphere of the standard - when stylus is oriented vertically; or hemisphere oriented at the appropriate angle in the case of different configurations of probe. Each measured point collected on the sphere has an individual approach vector directed from the center of the calibration ball to the nominal measured point. The value of this vector is determined by comparing the result of measured distance between the calibration ball and tip ball with nominal value, which is the sum of the radii 
of the applied balls. The difference is the measurement error associated with the direction with which the point measurement was taken. Since it is assumed that the calibration ball is perfect - the shape deviation equals to zero - the error in a given direction is assigned to the tip ball and compensated during further measurements.

To ensure the maximum possible accuracy of the machine, the qualification process should be repeated as often as necessary, especially in case of any changes make in machine configuration, such as the probe geometry, probes exchange, and even after removing and re-attaching the probe to the head. To maintain this principle in industrial conditions, it would be necessary to perform a calibration process couple times a day. Unfortunately, most often in practice, despite the relatively short qualification time, the qualification is performed less often what can result in reduction of measurement accuracy.

\section{QUALIFICATION OF FIVE-AXIS COORDINATE MEASURING MACHINES}

Five-axis measuring systems which utilize the articulating probe heads introduce interesting possibilities for Coordinate Metrology. Essential part of such systems is probe head which can rotate about mutually orthogonal axes (vertical axis is called "B", horizontal axis is called "A"). Of course articulating probes have been used in CMMs for many years, but usually they had limited range of angular orientation that can be obtained (for example they can rotate with step equal to $15^{\circ}$ around vertical axis) and each utilized orientation need to be calibrated separately. During points measurement probe orientation is fixed and can be changed only between actual measurements. The five-axis measuring systems allow to perform measurements using the head rotations, and what is even more important, in some cases measurements can be done even without CMM's moves (for example circular features inspection). Such an approach leads to acceleration of measurement process because the machine units, which are often made of heavy materials, do not need to be accelerated and decelerated for each measuring point. Research described in [2] shows that five-axis measurement may reduce measurement duration up to half of the time needed for standard three axis measurements, without significant loss in accuracy or even offering its improvement.
The continuous indexation of the discussed probes requires special qualification process. Normally, in case of articulating probes with fixed indexation, the head geometrical errors have the same impact on all points measured with certain probe orientation, moreover, qualification takes the gravitational drop of the probe into account. In five-axis measuring systems the orientation of probe used for measurements is unlimited (in measuring volume of head) and may vary largely between the subsequent points measurements as well as the influence of the above-mentioned errors sources on measurement result. Therefore, the qualification process for probe heads used in five-axis measuring systems has to be done in such a way to consider changes in probe performance, depending on its orientation utilized for each measurement point. Generally, qualification process for such heads is divided into two separate procedures, both based on the measurements of reference calibration sphere. Firstly, the geometrical calibration of probe head is done by comparing the results of sphere measurements performed mostly with head rotational movements, obtained for different angular orientations of probe. During this step the head works with the so called "Reference Tool" (Reference Probe) which should be used only for qualification purposes. In the second stage the appropriate configuration of probe is qualified. This part involves such step as: probe qualification in threeaxis mode with head angles equal to $\mathrm{A}=0, \mathrm{~B}=0$ and $\mathrm{A}=0, \mathrm{~B}=180$; determination of optimal probe head acceleration (done without sphere measurements); determination of optimal speed for probe head rotational measurements, determination of probe installation parameters, determination of interpolated probe indications map. The last three are based on the measurements of sphere in different cross sections using different orientations of probe, mostly with rotational moves of probe head. Both head and probe qualifications are rather time consuming (taking more than 10 minutes), but they do not have to be repeated until new probe configuration is needed or the probe head would be disassembled.

\section{EXPERIMENT AND RESULTS}

To assess the influence of qualification on the performance of articulating probe heads used in five-axis measuring systems authors decided to perform a series of measurements of the refer- 


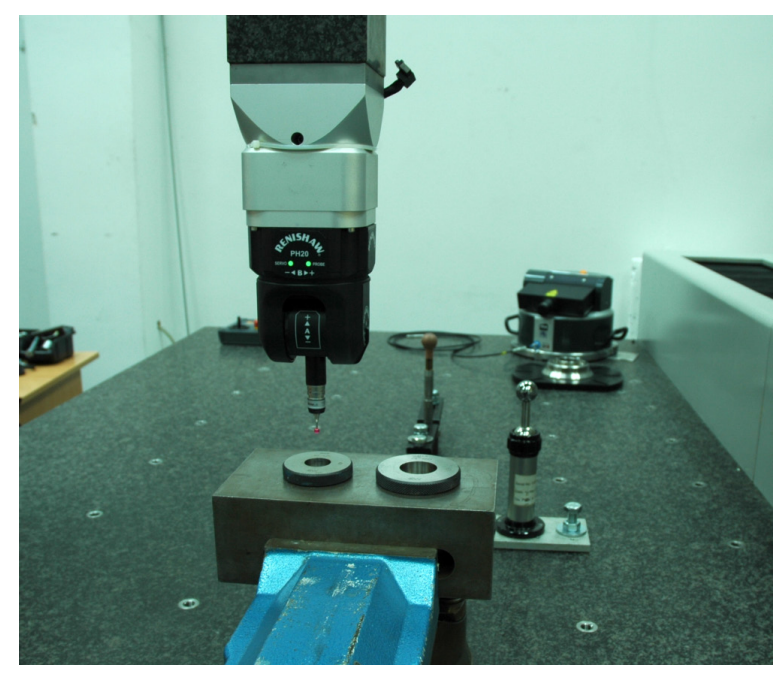

Fig. 1. The research station - reference ring and rings used for qualifications on the Zeiss WMM 850S

ence objects but each time preceded by different qualification processes. The machine used in described experiments is Zeiss WMM850S, equipped with Renishaw $\mathrm{PH} 20$ probe head, with touch trigger TP20 probe. The utilized tip ball diameter is $4 \mathrm{~mm}$ while stylus length is $12 \mathrm{~mm}$. The research station is shown in (Fig. 1). The measurements were done using Modus software. It was decided to use two calibration balls - the standard sphere and the ball with a large shape deviations which influence qualification process should be clearly visible. Both spheres have been calibrated in the Laboratory of Coordinate Metrology (LCM) on the Leitz PMM 12106 reference machine. The better one is characterized by the form deviation of $0.0004 \mathrm{~mm}$ and further will be named "SPH CAL". The second ball has form deviation of $0.11157 \mathrm{~mm}$ and will be marked as "SPH PLA". Both spheres have similar diameters equals about $25 \mathrm{~mm}$.

The four cases have been examined. The qualification marked as "PH_OK_TP_OK" means that both the head qualification and probe qualification were done using SPH CAL reference ball. The "PH_NOK_TP_NOK" means that both qualifications were performed using the SPH PLA sphere. In two remaining cases one qualification was done using SPH CAL while second utilizing SPH PLA. "PH_NOK_TP_OK" means that head qualification was done using SPH PLA while probe qualification using SPH CAL, and "PH_OK_TP_NOK" means the opposite eventuality. In all qualifications the same stylus and tip ball were used. After each considered qualification the reference objects were measured ten times. They include the reference ring of $20 \mathrm{~mm}$ diameter and reference sphere of $25 \mathrm{~mm}$ diameter. Both objects are characterized by negligible shape errors. The sphere was measured on its equator in 20 evenly distributed points using rotational movements of probe head (S25) and in standard three axis mode (S25 3AXI). " $\mathrm{R} 20 \mathrm{z} 90$ " is the $\mathrm{R} 20$ ring rotated $90^{\circ}$ along $\mathrm{z}$ axis of the machine in counter clockwise direction. This kind of mounting of the standard ring was used in order to prove that the measurement errors should be attributed to the functioning of the probe head, not to the shape of the ring itself. In that situation, the obtained characteristic of errors should remain the same as before the rotation. The ring measurements were done also in two configurations but each time using only rotational moves of head. The strategy involves measurement of 20 points distributed evenly on the middle of ring height. Firstly, the ring was fixed in machine volume in such a way that its main axis would be parallel to the $\mathrm{z}$ axis of machine (R20). For such orientation of measured feature the default measurement algorithm was designed by producer, so that all points are measured with the same TP20 deflection because head rotates mostly about vertical axis while A angle is almost the same each time. Such a solution is beneficial regarding measurement accuracy, however, it is only one of countless possible orientations which can be found in metrological practice. That is why measurements were repeated for different orientation of the ring, in which the main axis of the ring is inclined at an angle of $30^{\circ}$ to the $\mathrm{XY}$ plane of machine (R20y30).

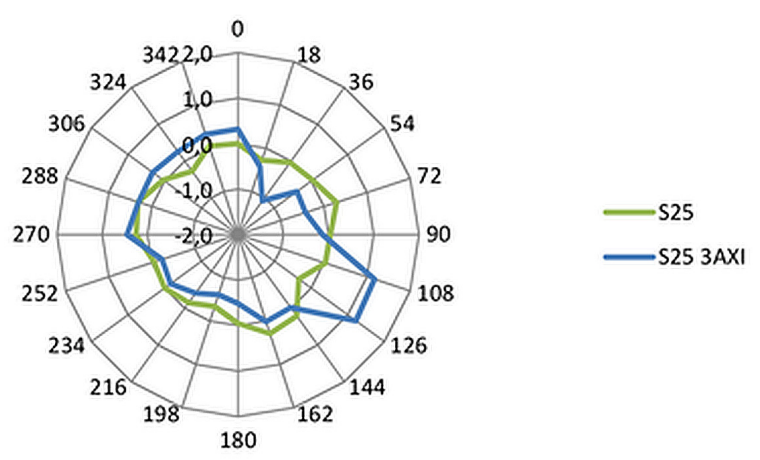

Fig. 2. Graphical comparison of the results of measurements of material standard obtained with five-axis measurements (S25) and three-axis measurements (S25 3AXI) for calibration "PH_OK_TP_OK". Values on vertical axis gives mean radial error in $\mu \mathrm{m}$, values on circumference of graph gives values of $\alpha$ angle in degrees 


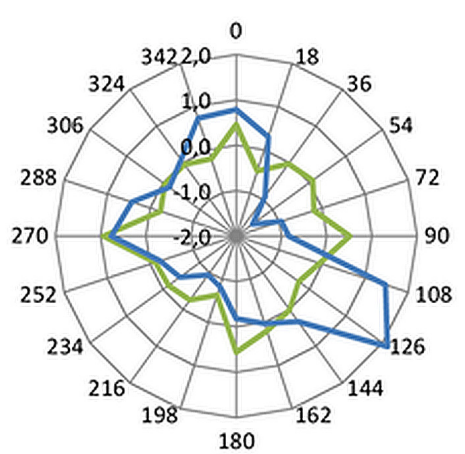

Fig. 3. Graphical comparison of the results of measurements of material standard obtained with five-axis measurements (S25) and three-axis measurements (S25 3AXI) for calibration "PH_NOK_TP_NOK". Values on vertical axis gives mean radial error in $\mu \mathrm{m}$, values on circumference of graph gives values of $\alpha$ angle in degrees

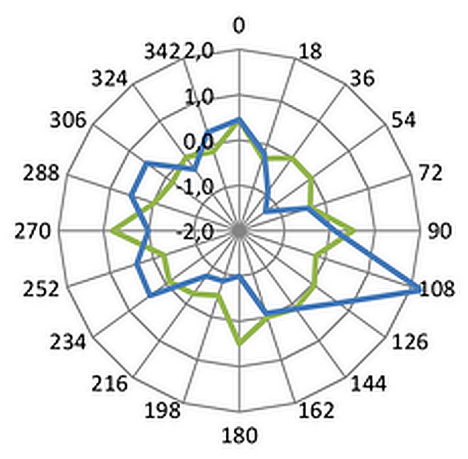

\section{$-\$ 25$}

- 253 3AXI

Fig. 4. Graphical comparison of the results of measurements of material standard obtained with five-axis measurements (S25) and three-axis measurements

(S25 3AXI) for calibration "PH_OK_TP_NOK".

Values on vertical axis gives mean radial error in $\mu \mathrm{m}$, values on circumference of graph gives values of $\alpha$ angle in degrees

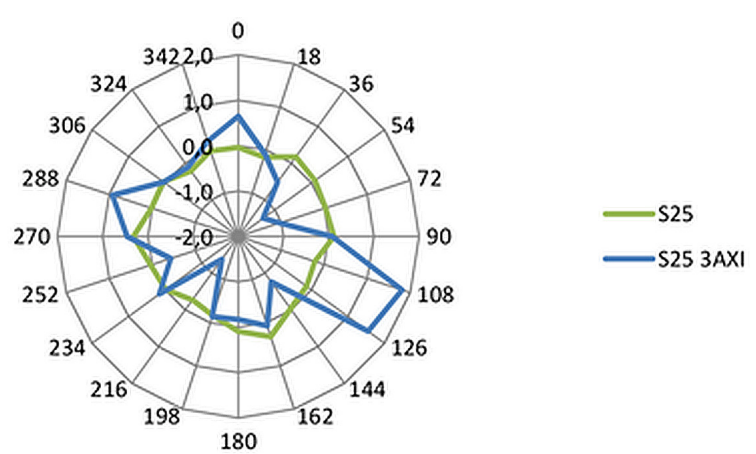

Fig. 5. Graphical comparison of the results of measurements of material standard obtained with five-axis measurements (S25) and three-axis measurements (S25 3AXI) for calibration "PH_NOK_TP_OK". Values on vertical axis gives mean radial error in $\mu \mathrm{m}$, values on circumference of graph gives values of $\alpha$ angle in degrees

The features that were under evaluation included: diameter and form errors of standard sphere $\mathrm{S} 25$, diameter and form errors of standard ring R20. Each time also the deviation maps given as radial errors in different directions were prepared and analyzed. The measurement was repeated 10 times for all the measured features. Then, the mean radial error for each measuring point was calculated as well as mean values of diameter and form errors. In all of the figures presented below (Fig. 2 $-5)$, the angular positions of points in degrees are given at the circumference of the graph, while the vertical axes present radial errors in $\mu \mathrm{m}$.

Below, the results of S25 sphere measurements performed using 5-axis mode were presented in Figure 6 (Fig. 6). The results of calibration of spheres (performed on PMM 12106

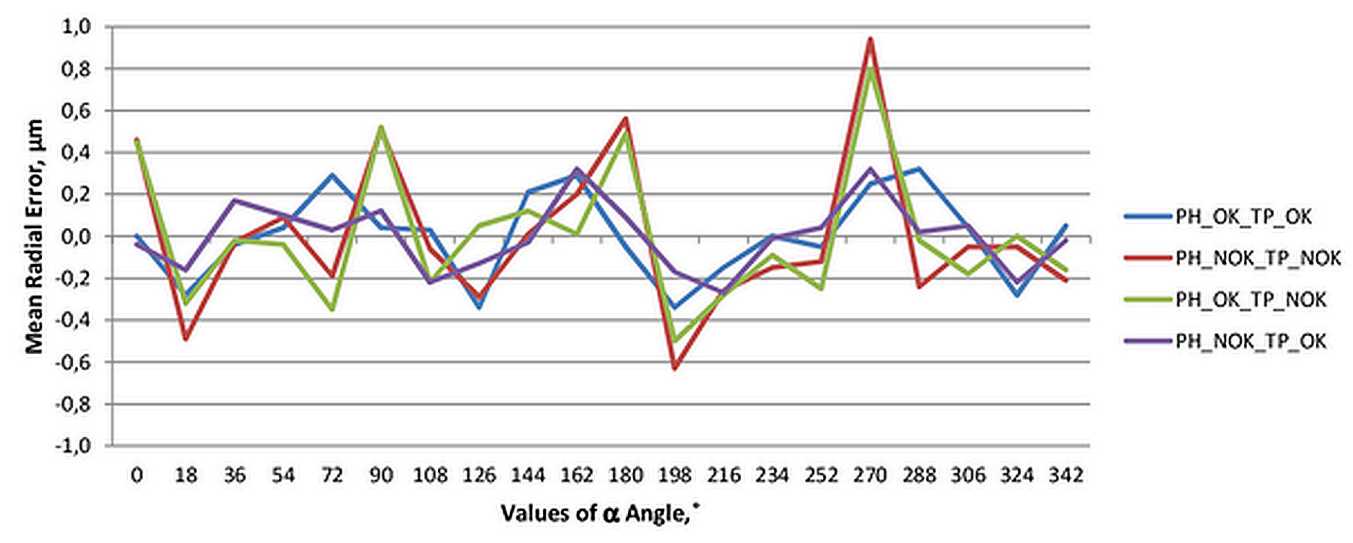

Fig. 6. Mean Radial Error in $\mu \mathrm{m}$, for different $\alpha$ values given in degrees ${ }^{\circ}$, obtained for five-axis measurements of S25 sphere for different qualification processes 


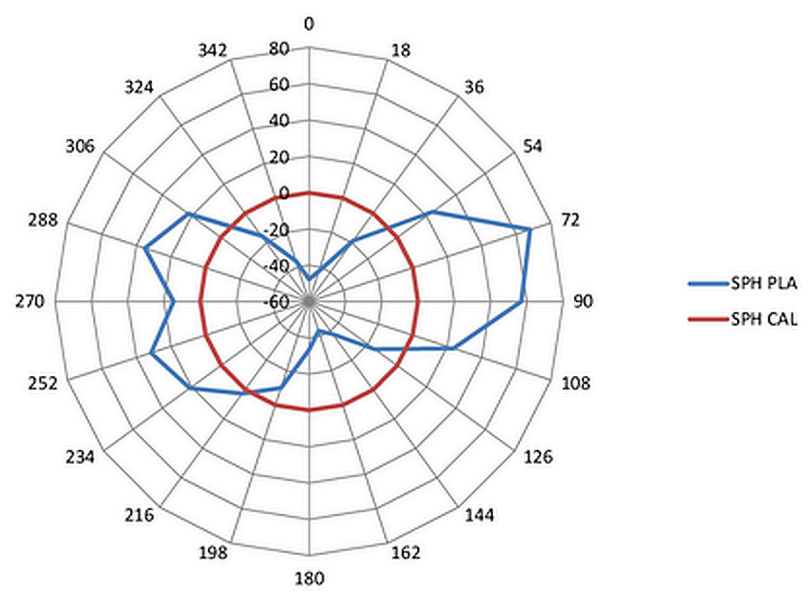

Fig. 7. Graphical comparison of the results of qualification spheres calibration. Values on vertical axis gives mean radial error in $\mu \mathrm{m}$, values on circumference of graph gives values of $\alpha$ angle in degrees

Table 1. Results obtained for „PH_OK_TP_OK” calibration. Diameter error means the difference between the measured diameter and nominal value of diameter obtained during standard calibration. For sphere nominal value equals to $25,0005 \mathrm{~mm}$, for ring $19,9997 \mathrm{~mm}$. All values given in $\mathrm{mm}$

\begin{tabular}{|c|c|c|c|c|c|}
\hline PH_OK_TP_OK & S25 & S25 3AXI & R20 & R20z90 & R20y30 \\
\hline Diameter & 24,9998 & 24,9986 & 20,0010 & 20,0013 & 20,0008 \\
\hline Diameter Error & $-0,0007$ & $-0,0019$ & 0,0013 & 0,0016 & 0,0011 \\
\hline Form Deviation & 0,0010 & 0,0027 & 0,0013 & 0,0010 & 0,0009 \\
\hline
\end{tabular}

Table 2. Results obtained for „PH_NOK_TP_NOK” calibration. Diameter error means the difference between the measured diameter and nominal value of diameter obtained during standard calibration. For sphere nominal value equals to $25,0005 \mathrm{~mm}$, for ring 19,9997 $\mathrm{mm}$. All values given in $\mathrm{mm}$

\begin{tabular}{|c|c|c|c|c|c|}
\hline PH_NOK_TP_NOK & S25 & S25 3AXI & R20 & R20z90 & R20y30 \\
\hline Diameter & 24,8911 & 24,9015 & 20,0500 & 20,0499 & 20,0537 \\
\hline Diameter Error & $-0,1094$ & $-0,0990$ & 0,0503 & 0,0502 & 0,0540 \\
\hline Form Deviation & 0,0016 & 0,0037 & 0,0035 & 0,0030 & 0,0044 \\
\hline
\end{tabular}

Table 3. Results obtained for „PH_OK_TP_NOK” calibration. Diameter error means the difference between the measured diameter and nominal value of diameter obtained during standard calibration. For sphere nominal value equals to $25,0005 \mathrm{~mm}$, for ring $19,9997 \mathrm{~mm}$. All values given in $\mathrm{mm}$

\begin{tabular}{|c|c|c|c|c|c|}
\hline PH_OK_TP_NOK & S25 & S25 3AXI & R20 & R20z90 & R20y30 \\
\hline Diameter & 24,8912 & 24,9001 & 20,0521 & 20,0522 & 20,0541 \\
\hline Diameter Error & $-0,1093$ & $-0,1004$ & 0,0524 & 0,0525 & 0,0544 \\
\hline Form Deviation & 0,0015 & 0,0036 & 0,0018 & 0,0012 & 0,0026 \\
\hline
\end{tabular}

Table 4. Results obtained for „PH_NOK_TP_OK” calibration. Diameter error means the difference between the measured diameter and nominal value of diameter obtained during standard calibration. For sphere nominal value equals to $25,0005 \mathrm{~mm}$, for ring $19,9997 \mathrm{~mm}$. All values given in $\mathrm{mm}$

\begin{tabular}{|c|c|c|c|c|c|}
\hline PH_NOK_TP_OK & S25 & S25 3AXI & R20 & R20z90 & R20y30 \\
\hline Diameter & 24,9993 & 24,9987 & 20,0008 & 20,0009 & 20,0015 \\
\hline Diameter Error & $-0,0012$ & $-0,0018$ & 0,0011 & 0,0012 & 0,0018 \\
\hline Form Deviation & 0,0010 & 0,0034 & 0,0026 & 0,0020 & 0,0036 \\
\hline
\end{tabular}




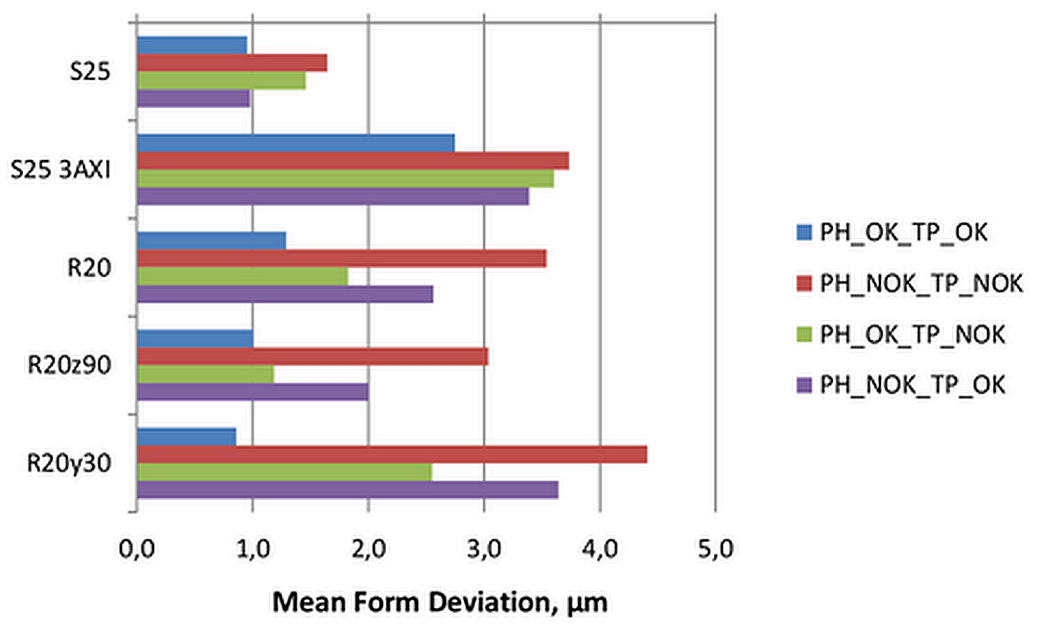

Fig. 8. Mean form deviation in $\mu \mathrm{m}$ obtained for reference objects measurement for used qualification processes

machine) that were used for qualification of head and probes were presented in figure 7 (Fig. 7). The summary of the obtained results was given in Tables 1 - 4. Graphical comparison of form errors obtained for sphere and ring standards using different qualifications of probe head and the probe was given in figure 8 (Fig. 8).

Analyzing the results of the performed experiments it is clearly visible that the influence of head and probe calibration results is bigger for the diameters of measured objects than for their form errors. The calibration of head and probe has minimal influence especially in the case of measurements of form errors performed in 5-axis mode for features oriented perpendicularly to XY plane of machine's coordinate system. Then, measurement of all points is done using the same contact point at the surface of probe tip. As was supposed, this approach makes accurate measurements of rotary workpieces possible, regardless of the results of probe and head calibration. However, the identification of dimensions of measured objects is influenced in higher level, because only one of the effective radii of probe tip determined during the probe calibration is used. It is the one corresponding to point (or to point laying in its closest proximity) on the tip surface which touch the measured object. So the error coming from probe calibration performed on improper material standard is repeated for all the points measured on the workpiece.

Because of relatively small values of differences between the results obtained using different types of calibrations for 5-axis mode the authors decided also to add measurements of sphere using classical 3-axis mode. Here, the differences were expected to be bigger. As the results show, it is not exactly true. The differences obtained for form errors were in fact bigger but their scale is much smaller than expected. The differences obtained for dimensions were even smaller for 3 -axis mode than for 5 -axis one.

Similar conclusions may be drawn from measurements of standard ring that was inclined in relation to base position (in which the axis of the ring is perpendicular to machine's table) that were done using 5-axis mode. They were performed in order to eliminate the strategy of measurement described in first paragraph of this section, so the measurements done using only one contact point. When the ring is inclined, it is impossible to use this strategy and the contact between probe tip and measured workpiece occurs in different points. In this case, the poor results of probe calibration coming from calibration sphere's form errors should be clearly visible in results of workpiece's form errors measurements, which should reflect the form deviations of calibration sphere. The obtained results are in contradiction to this statement. The differences between the results obtained for base position and inclined position are negligible. The maximum difference in results of form errors for different combinations of $\mathrm{PH}$ and $\mathrm{TP}$ being $\mathrm{OK} / \mathrm{NOK}$ is equal to $0,0035 \mathrm{~mm}$ despite the fact that there is about 0,1 $\mathrm{mm}$ difference in form errors of spherical standards used for performing head and probe calibration.

\section{CONCLUSIONS}

The results of the performed experiments showed that it is the probe calibration (denoted in results section as "TP") that influence the measurements of dimensions, while head calibration 
(denoted in results section as " $\mathrm{PH}$ ") does not have that big influence. It is understandable, as the head calibration is done mainly to determine the correction matrix for the $\mathrm{PH} 20$ probe head while the probe calibration aims in finding of actual parameters of the probe and its tip.

The final conclusion coming from the presented experiments is that the form errors values attributed to the spherical standard that was used for head and probe calibration has a minor impact on the results of workpiece's form errors measurements. The authors suppose that the source of such findings may be identified as existence of internal algorithms used in the head and probe calibration processes in the UCC Assist and UCC Server software developed by probe head's producer. These algorithms are know-how of the probe head's producer and are not publicly available for the analysis.

\section{ACKNOWLEDGMENTS}

Reported research was realized as part of a project financed by National Science Centre, Poland, grant no. 2015/17/D/ST8/01280.

\section{REFERENCES}

1. Cuesta E., Alvarez B., Sanchez-Lasheras F., Gonzalez-Madruga D.: A statistical approach to prediction of the CMM drift behaviour using a calibrated mechanical artefact, Metrology and Measurement Systems, 22 (3), 2015, 417-428.
2. Gruza M., Gąska A., Gąska P., Harmatys W., Sładek J.: Analiza wpływu trybu pracy pięcioosiowego systemu współrzędnościowego na dokładność pomiaru. Mechanik, 2016, 11, 1708 - 1709.

3. Harmatys W., Gąska P., Gąska A., Gruza M., Sładek J., Determination of temperature impact on CMM residual kinematic errors distribution, Advances in Science and Technology Research Journal, 9 (26), 2015, 113-117.

4. Hasselman T., Lloyd G.: A top-down approach to calibration, validation, uncertainty quantification and predictive accuracy assessment, Computer Methods in Applied Mechanics and Engineering 197 (29-32), 2008, 2596-2606.

5. Hocken R. J., Pereira P. H.: Coordinate Measuring Machines and Systems, Second Edition. CRC Press, USA, 2012.

6. Ratajczyk E., Woźniak A.: Współrzędnościowe systemy pomiarowe. Oficyna Wydawnicza Politechniki Warszawskiej, Polska, Warszawa 2016.

7. Sładek, J: Coordinate Metrology: Accuracy of Systems and Measurements. Springer. Germany, Berlin 2016.

8. Weckenmann A., Estler T., Peggs G., McMurtry D.: Probing Systems in Dimensional Metrology. CIRP Annals-Manufacturing Technology, 2004, 53(2), 657-684.

9. Zavvar M, Ramezani F, A method based on fuzz system for assessing the reliability of software based aspects, Advances in Science and Technology Research Journal, 9 (27), 2015, 143-148. 